\title{
Crescimento do guanandi e produção de mandioca e araruta em sistemas agroflorestais
}

\section{Growth of guanandi and cassava and arrowroot production in agroforestry systems}

\author{
Antonio Carlos Pries Devide ${ }^{\left(\mathbb{D}^{1}\right.}$; Cristina Maria de Castro ${ }^{\mathbb{D}^{2}}$; Raul de Lucena Duarte Ribeiro ${ }^{3}{ }^{3}$
}

${ }^{1}$ Engenheiro Agrônomo, Doutor em Fitotecnia - Agroecologia, Pesquisador da Agência Paulista de Tecnologia dos Agronegócios, Pindamonhangaba, São Paulo. Fone: (12) 3642-1823; antoniodevide@ apta.sp.gov.br; ${ }^{2}$ Engenheira Agrônoma, Doutora em Ciência do Solo, Pesquisadora da Agência Paulista de Tecnologia dos Agronegócios; cristinacastro@apta.sp.gov.br; ${ }^{3}$ Engenheiro Agrônomo, Doutor em Fitopatologia, Professor associado do Programa de Pós-graduação em Fitotecnia do Instituto de Agronomia da Universidade Federal Rural do Rio de Janeiro, raulucena @ gmail.com

\section{A R T I G O}

Recebido: 22/01/2018

Aprovado: 10/03/2019

Palavras-chave:

Transição agroecológica

Agrofloresta

Restauração ambiental

Alterações climáticas

\section{Key words:}

Agroecological transition

Agroforestry

Environmental restoring

Climate change

\section{R E S U M O}

No Vale do Paraíba do Sul, entre os estados do Rio de Janeiro e São Paulo, a substituição da Mata Atlântica para o cultivo do café degradou os solos e os cursos d'água. O cultivo de espécies florestais pode ajudar a restaurar esse ambiente. O guanandi (Calophyllum brasiliense Cambess.) é uma espécie florestal nativa com grande potencial de exploração econômica da madeira. O seu cultivo em sistema agroflorestal - SAF pode tornar-se mais vantajoso devido o seu moderado crescimento. Os objetivos com essa pesquisa foram comparar o crescimento do guanandi em monocultura e na diversificação agroflorestal em SAFs simples e biodiverso, assim como avaliar o desempenho da mandioca (Manihot esculenta Crantz.) e araruta (Maranta arundinacea L.) nesses sistemas, em ciclos com alta precipitação pluvial e escassez hídrica. O SAF biodiverso foi acrescido de feijão guandu, bananeira, palmeira juçara e diversidade arbórea. Os sistemas agroflorestais não prejudicaram o crescimento do guanandi, que incrementou a circunferência a altura do peito no SAF simples após três anos de conversão. As culturas alimentares de mandioca e araruta apresentaram desempenho compatível com a densidade de plantio utilizada nos SAFs, com produções significativamente superiores no SAF simples. A araruta suportou o estresse ambiental em ambos SAFs e dispensa o replantio, ocupando naturalmente o sub-bosque. $\mathrm{O}$ SAF simples é um sistema para agricultores interessados em obter o máximo rendimento de cultivos anuais em associação com a espécie florestal. O SAF biodiverso é adequado para diversificar a produção agrícola e restaurar o ambiente concomitantemente.

\section{A B S T R A C T}

In the Paraíba do Sul Valley, between the states of Rio de Janeiro and São Paulo, the replacement of the Atlantic Forest for coffee cultivation has degraded soils and watercourses. Growing forest species can help restore this environment. The guanandi (Calophyllum brasiliense Cambess.) is a native forest species with great potential for economic exploitation of wood. Its cultivation in agroforestry system - AFS can become more advantageous due to its moderate growth. The objectives of this research were to compare the growth of guanandi in monoculture and agroforestry diversification in simple and biodiverse AFSs, as well as to evaluate the performance of cassava (Manihot esculenta Crantz.) and arrowroot (Maranta arundinacea L.) in these systems, in cycles with high rainfall and water scarcity. The biodiverse AFS was added with pigeon pea, banana, juçara palm and arboreal diversity. Agroforestry systems did not affect the growth of guanandi, which increased the circumference of chest height in simple AFS after three years of conversion. Cassava and arrowroot food crops presented performance compatible with the planting density used in AFSs, with significantly higher yields in simple AFS. Arrowroot has withstood environmental stress on both AFSs and does not require replanting, naturally occupying the understory. Simple AFS is a system for farmers interested in obtaining the maximum yield of annual crops in association with the forest species. The biodiverse AFS is appropriate to diversify agricultural production and restore the environment concomitantly.

\author{
Revista Verde \\ ISSN 1981-8203 \\ Pombal, Paraíba, Brasil
}

v. 14, n.2, abr.-jun, p.303-311, 2019 doi: 10.18378/rvads.v14i2.6306 


\section{INTRODUÇÃO}

Globalmente, mais de dois bilhões de hectares de áreas degradadas e desflorestadas são oportunidades para se trabalhar a restauração com florestas plantadas e sistemas agroflorestais (SAFs) (LAESTADIUS et al., 2011). O plantio de florestas comerciais com espécies nativas é uma boa opção de investimento de médio a longo prazo. A diversificação dos plantios florestais de espécies de crescimento lento com SAFs, como no caso do guanandi (Calophyllum brasiliense Cambess.), pode ajudar a recuperar paisagens degradadas e mitigar os efeitos das alterações do clima. Isto, por meio do controle da erosão, aumenta a fertilidade dos solos e a disponibilidade da água, reforça a segurança alimentar e a nutrição com a produção de variedades de alimentos, forragens e produtos florestais (ALTIERI; NICHOLLS, 2017; FAO, 2017; LAUDARES et al., 2017; LUEDELING et al., 2014).

A consorciação de cultivos agrícolas e florestais não é recente (ABDO et al., 2012; DARONCO et al., 2012; MEDEIROS et al., 2015). Mas, o plantio de enriquecimento realizado em áreas de monocultura florestal para acelerar a restauração ambiental ainda foi pouco estudado (RAPPAPORT; MONTAGNINI 2014). Na restauração florestal sucessional com SAFs, aproveita-se a entrada de luz consorciando árvores e arbustos com cultivos agrícolas de maneira simultânea ou em sequência temporal (BRANCALION et al., 2012). O alto nível de biodiversidade dos SAFs melhora o desempenho agrícola e a resiliência do sistema às condições de seca extrema, que ocorreram nas duas últimas décadas nas regiões tropicais (BASCHE; EDELSON, 2017).

A cultura da mandioca (Manihot esculenta Crantz.) é a base para garantia da segurança alimentar para a agricultura familiar e culturalmente arraigada na agricultura brasileira, já pesquisada nos estágios iniciais dos SAFs e em reflorestamentos na restauração ambiental (DARONCO et al., 2012; SANTOS et al., 2002; SILVA et al., 2014). A araruta (Maranta arundinacea L.) é uma importante planta herbácea medicinal multiuso para agricultura familiar (KUMALASARI et al., 2012; SEIFERT; WATZL, 2007). Produz rizomas que estocam amido e também é forrageira (ROHANDI et al., 2017; SHINTU et al., 2015). Apesar de perene e tolerante ao sombreamento (ODEKU, 2013; SWADIJA et al.; 2013), a araruta foi pouco pesquisada em cultivo comercial em SAFs
(SWADIJA et al., 2013), reflorestamentos comerciais e na restauração florestal.

Os SAFs estão ganhando reconhecimento por compatibilizar a exploração agrícola com a restauração de florestas nativas (RAPPAPORT; MONTAGNINI, 2014). O estudo do plantio de enriquecimento do monocultivo de guanandi com SAFs se justifica com o aumento de renda ao produtor rural, pela restauração da paisagem, redução de perda de solo e proteção de áreas sensíveis à infiltração de água no solo. Devido o moderado crescimento do guanandi; que leva muitos anos para o aproveitamento da madeira; ao integrar a espécie perene lenhosa com culturas agrícolas, em arranjos espaciais e temporais, os SAFs se tornam uma importante categoria de fomento à restauração da paisagem e da cobertura florestal, além de gerar renda ao produtor rural (Secretaria do Meio Ambiente, 2018). A hipótese desse trabalho é de que ao menos um modelo de SAF gere produtos comerciais e promova a conservação da biodiversidade, sem que isto prejudique o crescimento do guanandi.

Os objetivos com essa pesquisa foram comparar o crescimento do guanandi em monocultura e na diversificação agroflorestal em SAFs simples e biodiverso, assim como avaliar o desempenho da mandioca e da araruta nesses sistemas.

\section{MATERIAL E MÉTODOS}

O experimento foi conduzido entre os anos de 2011 e

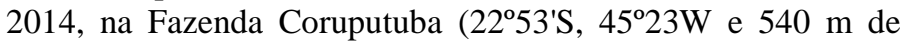
altitude), em Pindamonhangaba, São Paulo, no vale do Rio Paraíba do Sul, em uma importante área de transição da Serra do Mar para a Serra da Mantiqueira. O plantio de guanandi em monocultivo foi realizado no ano de 2008 no espaçamento 3,0 x $2,0 \mathrm{~m}$ em terraços fluviais e abrangeu áreas ciliares consideradas como de uso consolidado pelo Código Florestal. Os solos foram classificados como Cambissolo Flúvico e Planossolo Háplico, ambos são distróficos de boa drenagem e textura argilo arenosa, com as seguintes características químicas: $\mathrm{pH}(\mathrm{H} 2 \mathrm{O})=5,7 ; \mathrm{H}+\mathrm{Al}=3,22 \mathrm{mg} \mathrm{dm}^{-3} ; \mathrm{P}=52,3 \mathrm{mg}$ $\mathrm{dm}^{-3} ; \mathrm{K}=19 \mathrm{mg} \mathrm{dm}{ }^{-3} ; \mathrm{Ca}=1,3 \mathrm{cmol}_{\mathrm{c}} \mathrm{dm}^{-3} ; \mathrm{Mg}=0,8 \mathrm{cmol}_{\mathrm{c}}$ $\mathrm{dm}^{-3}$; matéria orgânica $=27,7 \mathrm{dag} \mathrm{dm}^{-3}$. O clima Cwa Subtropical de inverno seco segundo a classificação de Köppen foi monitorado (Tabela 1).

Tabela 1. Resenha agrometeorológica de Pindamonhangaba, São Paulo

\begin{tabular}{|c|c|c|c|c|}
\hline Resenha Agrometeorológica & 2011 & 2012 & 2013 & 2014 \\
\hline Dias de Chuva & 115 & 116 & 117 & 83 \\
\hline Chuva Total & 1307 & 1497 & 1158 & 619 \\
\hline Média Mensal da Chuva Total & 109 & 125 & 97 & 55 \\
\hline Temperatura Média Diária & 22,3 & 22,9 & 21,8 & 22,4 \\
\hline Temperatura Mínima Média Diária & 16,5 & 17,1 & 16,3 & 15,9 \\
\hline Temperatura Máxima Média Diária & 28,0 & 28,7 & 27,4 & 29,0 \\
\hline Dias com Temperatura Máxima de $32{ }^{\circ} \mathrm{C}$ ou maior & 6,4 & 8,2 & 4,9 & 9,0 \\
\hline Dias com Temperatura Máxima de $34{ }^{\circ} \mathrm{C}$ ou maior & 2,3 & 3,4 & 1,3 & 4,5 \\
\hline Dias com Temperatura Mínima de $13{ }^{\circ} \mathrm{C}$ ou abaixo & 7,3 & 6,6 & 7,1 & 9,2 \\
\hline
\end{tabular}

Fonte: CIIAGRO (2014).

Em julho de 2011, foi realizado o plantio experimental de enriquecimento com dois SAFs (simples e biodiverso) em delineamento em blocos ao acaso, com oito repetições em parcelas de $144 \mathrm{~m}^{2}$, constituídas de quatro linhas com seis árvores de guanandi em cada linha. Nas entrelinhas foram cultivadas as espécies alimentícias anuais em SAF simples, mandioca e araruta em rotação; e SAF biodiverso, que também 
foi enriquecido com feijão guandu (Cajanus cajan (L.) Millsp.), bananeira (Musa sp.), palmeira juçara (Euterpe edulis Mart.) e 14 espécies florestais nativas (Bixa orellana L., Pseudobombax grandiflorum (Cav.) A.Robyns, Schinus terebinthifolius Raddi., Anadenanthera colubrina (Vell.) Brenan, Inga vera Willd., Erythrina verna Vell, Croton floribundus Spreng., Joannesia princeps Vell., Zanthoxylum rhoifolium Lam., Handroanthus umbellatus (Sond.) Mattos, Handroanthus impetiginosus (Mart. ex DC.) Mattos, Magnolia ovata (A.St.-Hil.) Spreng, Citharexylum myrianthum Cham., Schizolobium parahyba (Vell.) Blake) alternadas $1,0 \mathrm{~m}$ entre si nas entrelinhas da cultura anual, entre as fileiras de guanandi.

No primeiro ciclo (2011-2012) de conversão agroflorestal comparou-se o desempenho da cultura da mandioca de mesa IAC 6-01, e nos dois ciclos seguintes avaliou-se a araruta em rotação. O plantio de mandioca foi realizado em cultivo mínimo em fileiras duplas $(0,80 \times 0,80 \mathrm{~m})$ equidistantes $1,1 \mathrm{~m}$ do guanandi. Recebeu calcário $\left(2,0 \mathrm{t} \mathrm{ha}^{-1}\right)$, composto orgânico $(11 \mathrm{t}$ $\left.\mathrm{ha}^{-1}\right)$, torta de mamona $\left(625 \mathrm{~kg} \mathrm{ha}^{-1}\right)$, farinha de ossos $(625 \mathrm{~kg}$ $\left.\mathrm{ha}^{-1}\right)$ e sulfato de potássio $\left(63 \mathrm{~kg} \mathrm{ha}^{-1}\right)$ em cobertura aos 45 dias após o plantio (DAP). No segundo ciclo (2012-2013) o cultivo intercalar da araruta 'Comum' foi realizado em plantio direto em fileiras duplas $(0,5 \times 0,8 \mathrm{~m})$ equidistantes $1,1 \mathrm{~m}$ do guanandi a partir de rizomas-sementes com $98 \mathrm{~g}$ de massa média, recebendo a mesma adubação da mandioca. No terceiro ciclo (2013-2014), subdividiu-se a parcela em faixas fixas comparando-se o replantio da araruta, realizado da mesma maneira que no ciclo anterior, com a brotação espontânea. Foram utilizados para o replantio os rizomas colhidos no SAF simples e ambos os tratamentos não foram adubados. O objetivo foi avaliar a viabilidade da exploração da araruta em manejo perene como vegetação típica nativa de sub-bosque em SAFs

$\mathrm{O}$ crescimento do guanandi em ambos os SAFs foi comparado ao monocultivo (controle), com avaliações realizadas com trena e fita métrica em cinco árvores demarcadas previamente nas fileiras centrais de cada parcela. Abrangeu altura total (do nível do solo até o topo da copa), raio da copa obtido pela média de duas medidas perpendiculares à linha de cultivo a partir da base do tronco à projeção lateral da copa; circunferência a altura do peito $(\mathrm{CAP}-1,30 \mathrm{~m})$ e circunferência à altura do solo (CAS), realizadas nos anos de avaliação (2011-2014). Da mandioca, determinou-se aos 10 meses de idade as produções total, descarte, comercial e aporte total de fitomassa (raízes, cepas, manivas e rama herbácea). A avaliação da araruta foi feita aos 10 meses de idade nos dois ciclos de produção, determinando-se a produção e o acúmulo de fitomassa fresca e seca (folhas e raízes).

Os dados do guanandi foram comparados pelo teste de Kruskall-Wallis $(\mathrm{p}<0.05)$. O desempenho da mandioca e da araruta foram comparados com o Teste Mann-Whitney U $(\mathrm{p}<0,05)$ e os efeitos na parcela sub-dividida da araruta com o Teste de Kruskal-Wallis $(\mathrm{p}<0,05)$. Foi utilizado o Programa STATSOFT (2004).

\section{RESULTADOS E DISCUSSÃO}

Durante todos os ciclos experimentais, que abrangeu um período de três anos de monitoramento do guanandi, verificouse similaridade de seu crescimento em monocultivo e nos SAFs, com significativo incremento da CAP do guanandi no SAF simples. Isto é visualizado na tabela 2, que apresenta o nível de significância das variáveis analisadas, e na figura 1, que contêm os valores numéricos de crescimento apresentados por meio de gráfico de barras.

Tabela 2. Nível de significância das variáveis biométricas do guanandi em monocultura, SAFs simples e biodiverso, pelo teste de Kruskall-Wallis $(\mathrm{p}<0.05)$

\begin{tabular}{lcccc}
\hline Avaliações & 2011 & 2012 & 2013 & 2014 \\
\hline Altura & 0,771 & 0,925 & 0,183 & 0,785 \\
CAP* & 0,925 & 0,797 & 0,295 & 0,033 \\
CAS* & 0,469 & 0,548 & 0,107 & 0,100 \\
Raio da copa & 0,920 & 0,728 & 0,432 & 0,100 \\
\hline
\end{tabular}

*CAP - -circunferência a altura do peito, CAS - circunferência a altura do solo

O lento crescimento do guanandi no estágio inicial resultou em espaços vazios, favorecendo a associação de espécies agrícolas e florestais em SAFs. Nos primeiros anos dos consórcios, essas espécies apresentaram tamanho reduzido em comparação ao porte do guanandi. Dessa maneira, pouco competiu com o guanandi por espaço e luz. Dentre os aspectos positivos que podem favorecer o crescimento do guanandi ao final do experimento, destacam-se os efeitos de adubações residuais e da reciclagem de nutrientes, por meio do aporte de resíduos orgânicos das espécies em associação; da ativação da biota do solo via micorrização da mandioca, araruta e guandu, espécie que também realiza a fixação biológica do nitrogênio.

Dentre os aspectos do crescimento do guanandi dos três para os seis anos de idade (Figura 1A) que favoreceram a consorciação em SAFs, destacam-se o incremento médio de altura de $321 \mathrm{~cm}$ para $617 \mathrm{~cm}$ e o raio da copa de $65 \mathrm{~cm}$ para $132 \mathrm{~cm}$ (Figura 1D). Os benefícios dessas associações para o crescimento do guanandi foram notáveis para a CAP que passou de 10,2 para 24,9 cm (Figura 1B) e menos pronunciado para a CAS de 19,5 $\mathrm{cm}$ para $42 \mathrm{~cm}$ (Figura 1C). Esse incremento do crescimento é considerado moderado e caracteriza a possibilidade de realizar cultivos e o plantio de enriquecimento de espécies agrícolas e florestais em consórcio.

Do ponto de vista silvicultural, o guanandi é uma árvore tardia de desenvolvimento lento quando comparada a outras espécies comerciais (BRENES; MONTAGNINI, 2006; PETIT; MONTAGNINI, 2006), o que possibilita associá-lo com outras espécies em SAFs; e do ponto de vista ambiental, é estratégica para restauração de áreas ciliares inundáveis (BRENES; MONTAGNINI, 2006; CARVALHO, 2003; DURIGAN; SILVEIRA, 1999). Nesse contexto, os SAFs podem se tornar um dos meios mais eficientes para se restaurar áreas ciliares com associações de espécies adaptadas, como a araruta. No Brasil, Durigan e Silveira (1999) registraram que o guanandi atingiu $4,34 \mathrm{~m}$ de altura aos nove anos de idade e superou as outras 16 espécies florestais de um total de 20 espécies que foram plantadas em associação parar restaurar uma mata ciliar em solos ácidos, com baixa fertilidade e elevado teor de alumínio. Na presente pesquisa, apesar da fertilidade do solo favorável ao crescimento do guanandi, houve restrição hídrica nos dois últimos anos resultando no porte intermediário aos verificados por Carvalho (2003), que registrou moderado crescimento em altura do guanandi $(781 \mathrm{~cm})$ e CAP $(31 \mathrm{~cm})$ em árvores de mesma idade em Manaus - AM em Latossolo 
Amarelo distrófico com condições hídricas e térmicas mais favoráveis ao seu crescimento; e em Paranaguá - PR, onde o guanandi atingiu apenas $468 \mathrm{~cm}$ de altura e $12 \mathrm{~cm}$ de CAP em Latossolo Vermelho-Amarelo distrófico (CARVALHO, 2003). Essa gradação no crescimento do guanandi entre regiões pode estar associada às diferenças climáticas em que foi cultivado. $\mathrm{O}$ maior crescimento foi verificado no clima Tropical úmido de monções com curto período seco em Manaus (região Norte); o crescimento intermediário ocorreu no clima Subtropical de inverno seco no Vale do Paraíba do Sul (região Sudeste) e o menor crescimento foi notado no clima Subtropical úmido mesotérmico em Paranaguá (região Sul). Com isso, depreendese que o guanandi possa apresentar maior crescimento em sítios com temperaturas mais elevadas.

Figura 1. Altura (A), circunferência a altura do peito - CAP (B), circunferência a altura do solo - CAS (C) e raio da copa do guanandi (D) em monocultivo, SAFs simples e biodiverso.
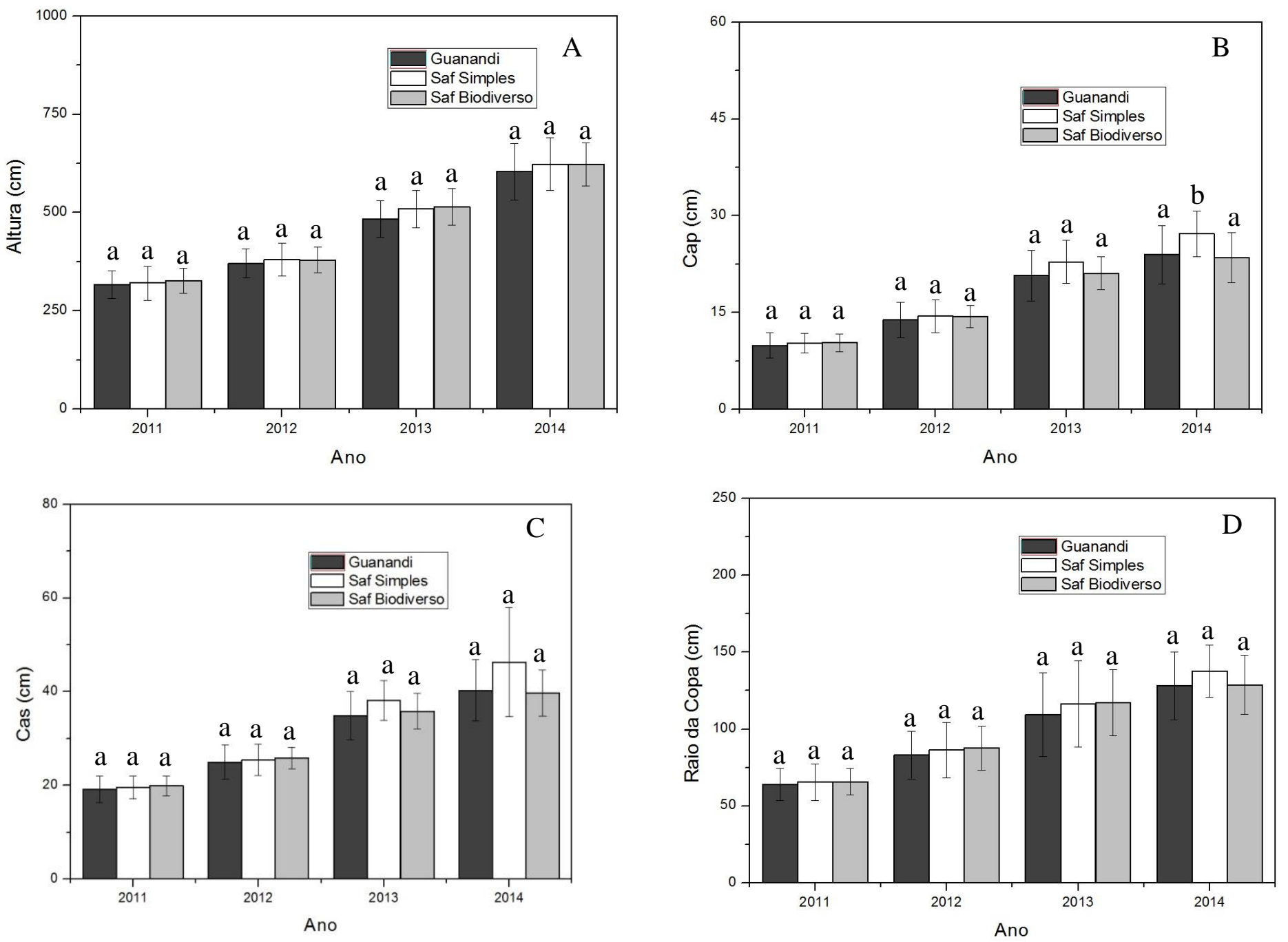

${ }^{1}$ Letras diferentes indicam diferenças significativas $(\mathrm{p}<0.05)$ pelo teste de Kruskal-Wallis.

O consórcio do guanandi com culturas alimentícias pode melhorar o aproveitamento da terra e reforçar a segurança alimentar sem prejudicar o seu crescimento. No estádio inicial da conversão, o guanandi foi associado com a mandioca IAC 601 ; esta sombreou o solo, controlou a vegetação espontânea indesejável nas entrelinhas (Figura 2A), acumulou abundante quantidade de fitomassa fresca (Figura 2B) e produziu raízes cozidas mais amarelas que as variedades comerciais, uma rica fonte de carotenoides pró-vitamínicos (Figura 2C).

O desempenho da mandioca foi satisfatório (Tabela 3), dada à baixa densidade de plantio (6.250 plantas $\left.\mathrm{ha}^{-1}\right)$ do arranjo agroflorestal. A mandioca produziu em media $6,2 \mathrm{t} \mathrm{ha}^{-1}$ de raízes comerciais, com oito raízes com massa média de 1,0 $\mathrm{kg}$ por planta (Figura 2B). O SAF simples foi o sistema que apresentou a maior produção da mandioca e isso é muito significativo para os agricultores. Entretanto, o SAF biodiverso possibilita a colheita futura de frutos de banana e juçara, que pode compensar a menor produção de mandioca. A adoção desse sistema por agricultores que desejam diversificar as fontes de renda e restaurar o ambiente, pode ser uma alternativa viável. Em outro estudo, Silva et al. (2014) obtiveram em cultivo intercalar com a cultura da banana a produção de $18,6 \mathrm{t} \mathrm{ha}^{-1} \mathrm{de}$ raízes de mandioca, no espaçamento de plantio 1,0 x $1,0 \mathrm{~m}$. Este valor é bem superior ao verificado no presente estudo em que havia o guanandi já desenvolvido. 
Figura 2. Mandioca nas entrelinhas do guanandi (A). Partição de fotoassimilados em 20 plantas (B). Raízes cozidas de mandioca IAC 6-01 ricas em betacaroteno (C).
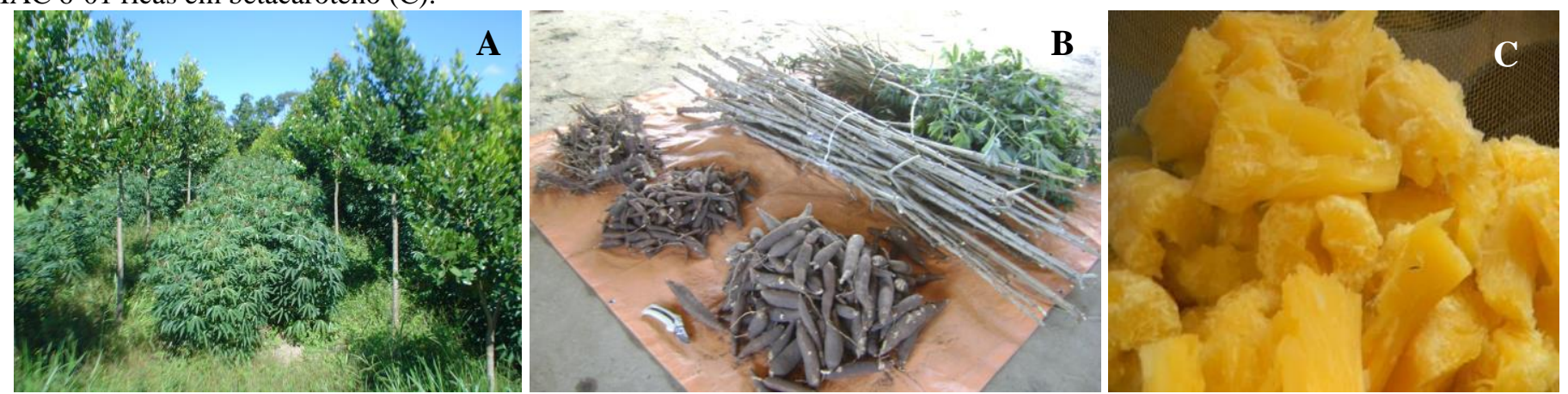

Fonte: Autor (2019)

Tabela 3. Desempenho da mandioca em SAFs simples e biodiverso com guanandi

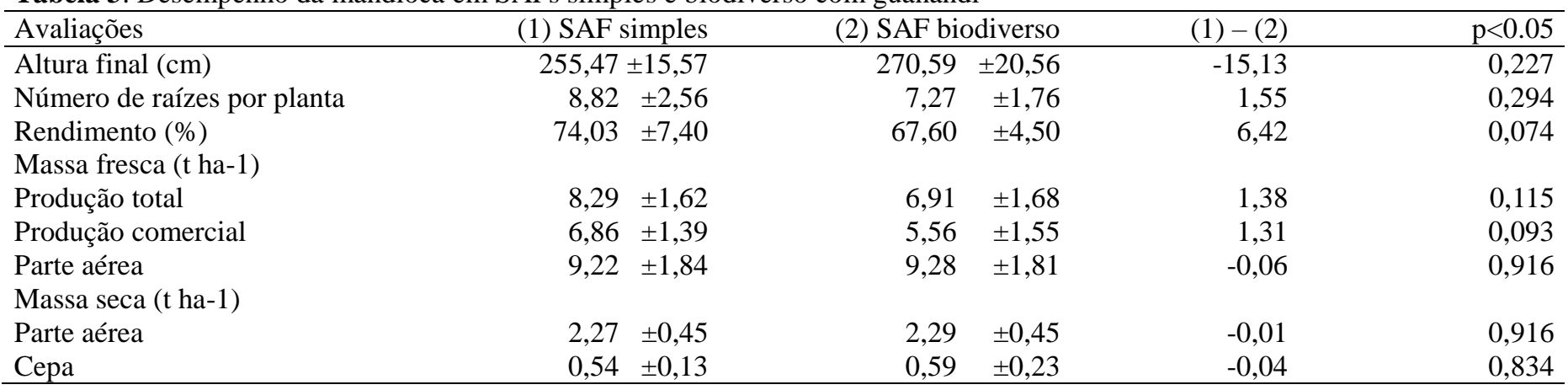

Daronco et al. (2012) avaliou o cultivo de mandioca associada com árvores nativas de reflorestamento em áreas ripárias no Oeste paulista, plantada no espaçamento $3,0 \times 0,5 \mathrm{~m}$ a mandioca produziu $6,1 \mathrm{t}$ ha-1 de raízes, valor similar ao obtido nesta pesquisa. $\mathrm{O}$ arranjo de plantio e a escolha da variedade correta interferem no desempenho da mandioca e a arquitetura das plantas é um fator a ser considerado na escolha da variedade para fins de consórcio, incluída nos estágios iniciais da restauração florestal (DARONCO et al., 2012; SANTOS et al., 2002) e nos SAFs (SILVA et al., 2014).

A arquitetura da variedade de mandioca IAC 6-01, que possui porte alto e ramificação alta e fechada a partir de $1,5 \mathrm{~m}$ de altura, facilitou os tratos culturais, favoreceu o consórcio de culturas e produziu cobertura do solo mais uniforme, que previne a erosão (DEVIDE et al., 2009). O rendimento da mandioca IAC 6-01 é considerado superior ao de variedades comuns e mais resistente às alterações do clima, além de conter elevado teor de vitamina $\mathrm{A}$ nas raízes de coloração mais amarelas que as variedades comerciais cultivadas no Brasil (FAPESP, 2012). Avaliada em monocultivo na densidade de 12.500 plantas ha-1, essa variedade produziu $17,5 \mathrm{t}$ ha-1 de raízes (CASTRO et al., 2009) e quando associada com adubos verdes aumentou a produção para $20 \mathrm{t}$ ha-1 (DEVIDE; CASTRO, 2010). O manejo orgânico da mandioca é estratégico para a transição agroecológica na agricultura familiar, por ser rústica e apresentar ciclo longo sem picos de absorção de nutrientes (DEVIDE; CASTRO, 2010). Como apenas 20\% de hastes são replantadas, restaram de 30 a $50 \%$ da fitomassa com alto valor nutricional (Figura 3B), que pode vir a melhorar a dieta de animais na forma de folhagem, feno ou silagem (MACEDO, 2016). Nos SAFs esses resíduos adicionados ao solo representaram o aporte médio de 9,2 t ha-1 de fitomassa, beneficiando a cultura subsequente.

Em rotação com a mandioca, a araruta introduzida em sistema de plantio direto apresentou desempenho superior no SAF simples, com produção de 7,6 tha-1 de rizomas e $15 \mathrm{t}$ ha- 1 de resíduos frescos (Tabela 4). Isto era esperado devido o alto nível de competição interespecífica, principalmente, com o crescimento da bananeira e das espécies arbóreas em associação no SAF biodiverso. Swadija et al. (2013) obtiveram a produção de 18,62 t ha-1 de rizomas frescos e 8,61 t ha-1 de massa seca, em associação de araruta com cultivo de coqueiros no espaçamento $30 \times 15 \mathrm{~cm}$, com plantas de $102 \mathrm{~cm}$ de altura, 1,5 brotos e 10 folhas por haste. O espaçamento utilizado por Swadija et al. (2013) é considerado adensado em comparação ao utilizado nessa pesquisa $(50 \times 80 \mathrm{~cm})$, o que justifica em parte a menor produção de araruta nos SAFs com guanandi. Porém, o maior crescimento vegetativo em altura da araruta e o número superior de brotos (9,8 folhas no SAF simples e 6,9 folhas por haste no SAF biodiverso) superaram as plantas de Swadija et al. (2013). Isto possivelmente indica que a araruta priorizou o desenvolvimento vegetativo em detrimento da produção de rizomas nos SAFs com guanandi. 
Tabela 4. Desempenho da araruta no SAFs simples e biodiverso com guanandi em dois ciclos de produção

\begin{tabular}{|c|c|c|c|c|c|}
\hline Análises & Unidade & (1) SAF simples & (2) SAF biodiverso & $(1)-(2)$ & $\mathrm{p}<0.05$ \\
\hline \multicolumn{6}{|l|}{ Ciclo 2012-2013 } \\
\hline MF Produção & tha-1 & $7,61 \pm 1,53$ & $1,92 \pm 0,74$ & 5,69 & 0,001 \\
\hline MF Resíduos & tha-1 & $15,03 \pm 2,37$ & $4,54 \pm 1,22$ & 10,49 & 0,001 \\
\hline MS Produção & tha-1 & $2,44 \pm 0,49$ & $0,61 \pm 0,24$ & 1,83 & 0,001 \\
\hline MS Resíduos & tha-1 & $3,19 \pm 0,50$ & $0,97 \quad \pm 0,26$ & 2,22 & 0,001 \\
\hline $\mathrm{N}^{\circ}$ folhas & por planta & $63,25 \pm 10,66$ & $23,25 \pm 6,80$ & 40,00 & 0,001 \\
\hline Altura & $\mathrm{cm}$ & $148,10 \pm 10,99$ & $127,90 \pm 17,22$ & 20,20 & 0,012 \\
\hline $\mathrm{N}^{\circ}$ brotações & por planta & $6,44 \pm 1,08$ & $3,38 \quad \pm 0,65$ & 3,06 & 0,001 \\
\hline $\mathrm{N}^{\circ}$ rizomas & por planta & $11,86 \pm 1,69$ & $3,84 \quad \pm 1,47$ & 8,02 & 0,001 \\
\hline \multicolumn{6}{|l|}{ Ciclo 2013-2014 } \\
\hline MF Produção & tha-1 & $2,51 \pm 1,09$ & $0,56 \pm 0,18$ & 1,95 & 0,004 \\
\hline MF Resíduos & tha-1 & $4,01 \pm 1,21$ & $0,99 \pm 0,24$ & 3,02 & 0,008 \\
\hline MS Produção & tha-1 & $0,90 \pm 0,39$ & $0,20 \pm 0,06$ & 0,70 & 0,008 \\
\hline MS Resíduos & tha-1 & $0,79 \pm 0,24$ & $0,20 \quad \pm 0,05$ & 0,59 & 0,008 \\
\hline $\mathrm{N}^{\circ}$ folhas & por planta & $45 \pm 8,42$ & $22 \pm 3,33$ & 23,00 & 0,461 \\
\hline Altura & $\mathrm{cm}$ & $127,69 \pm 3,06$ & $94,72 \pm 10,35$ & 32,97 & 0,001 \\
\hline $\mathrm{N}^{\circ}$ brotações & por planta & $5,16 \pm 1,10$ & $3,34 \pm 0,69$ & 1,82 & 0,001 \\
\hline $\mathrm{N}^{\circ}$ rizomas & por planta & $7,38 \quad \pm 1,85$ & $4,13 \pm 0,99$ & 3,25 & 0,002 \\
\hline
\end{tabular}

*MF - massa fresca, MS - massa seca.

Em monocultivo comercial, no espaçamento $0,33 \times 0,20$ $\mathrm{m}$, Zárate e Vieira (2005) obtiveram $14 \mathrm{t}$ ha-1 de rizomas de araruta. Comparando os dados da presente pesquisa com outros estudos, verifica-se que no SAF simples a produção por planta foi de $690 \mathrm{~g}$, valor superior ao obtido por Zárate e Vieira (2005) e Moreno et al. (2017), respectivamente, de $140 \mathrm{~g}$ e $330 \mathrm{~g}$ por planta. A araruta incrementa a produção por planta nos maiores espaçamentos (MORENO et al., 2017) mas os aumentos de produção são devidos ao maior número de plantas por hectare (ZÁRATE et al., 2012). Os efeitos na redução da produção por unidade de área é devido à escolha do espaçamento mais amplo no consórcio com árvores de guanandi de quatro anos de idade (Figura 4A) e com maior densidade de espécies no SAF biodiverso (Figura 4B), que ocasionaram maior sombreamento e o consequente desempenho superior da araruta no SAF simples. Além disso, o elevado aporte de resíduos da parte aérea significa um crescimento exuberante, que é um indicativo de que as plantas podem ter retardado a maturidade e o início do processo de senescência das folhas, com diminuição da translocação dos fotoassimilados de reserva para os órgãos armazenadores (ZÁRATE; VIEIRA, 2005).

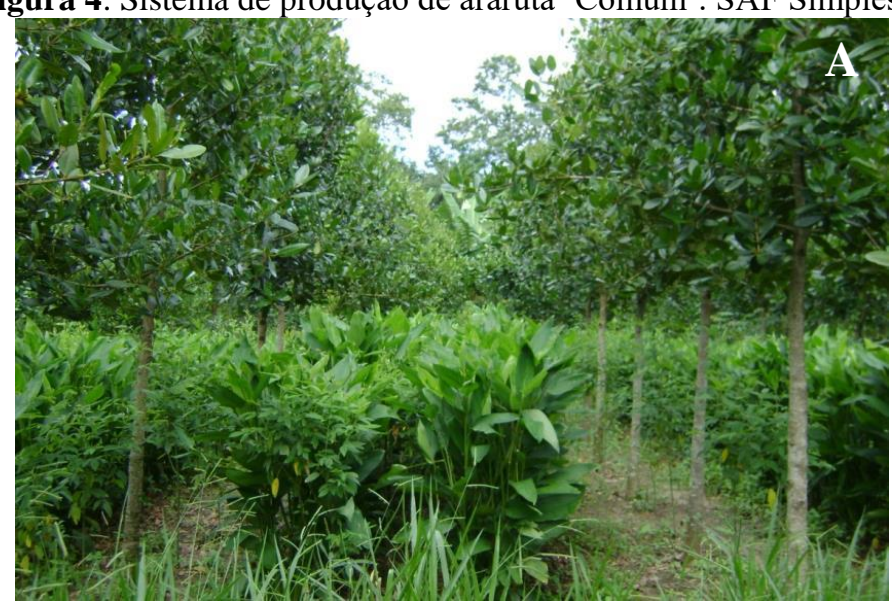

Fonte: Autor (2019)

No ciclo seguinte, a araruta se desenvolveu durante a maior estiagem dos últimos 80 anos que abrangeu toda a região do Vale do Paraíba do Sul (TARGA; BATISTA, 2015). Essas alterações climáticas foram caracterizadas pelo estresse hídrico e térmico, com menor número de dias de chuva e aumento da amplitude térmica (Tabela 1). Ao todo, choveu $454 \mathrm{~mm}$ no período de cultivo da araruta, sendo $318 \mathrm{~mm}$ do plantio ao mês

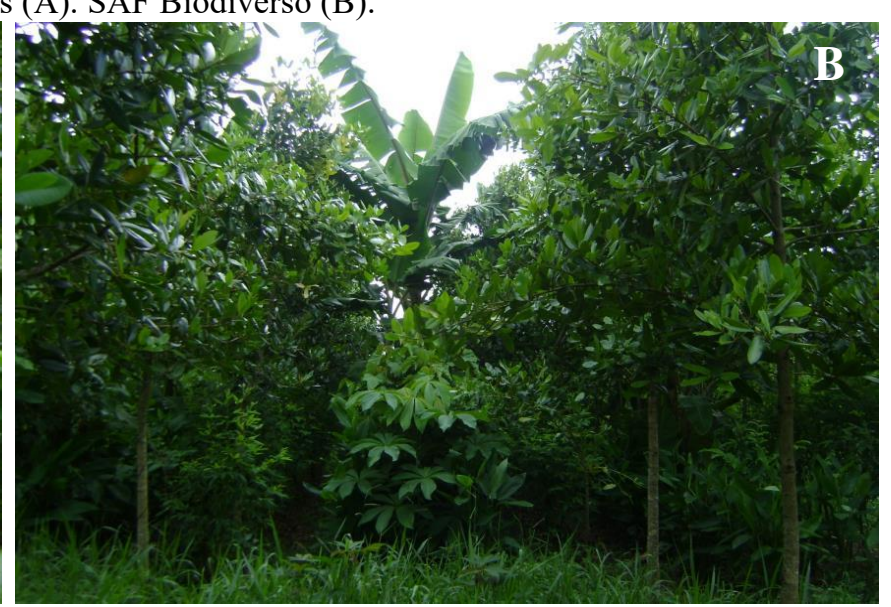

de março de 2014 e $136 \mathrm{~mm}$ até a colheita, com maior número de dias com temperatura máxima superior a $32{ }^{\circ} \mathrm{C}$ e temperatura inferior a $13{ }^{\circ} \mathrm{C}$. Os fatores ambientais limitaram a produção de rizomas, mas, ainda assim, as plantas atingiram $128 \mathrm{~cm}$ de altura com cinco brotações e sete rizomas, com produção em base fresca de 2,5 t ha-1 de rizomas e $4 \mathrm{t}$ ha-1 de fitomassa aérea no SAF simples (Tabela 4). A capacidade de 
sobrevivência da araruta em condições ambientais desfavoráveis caracteriza a sua rusticidade.

Ao comparar o desempenho da araruta proveniente de propagação espontânea e do replantio de rizomas-semente, verifica-se uma similaridade entre os sistemas de plantio dentro de cada SAF (Tabela 5).

Tabela 5. Diferenças entre araruta replantada e espontânea no SAF simples e biodiverso com guanandi

\begin{tabular}{|c|c|c|c|c|c|c|c|}
\hline Tratamentos & Altura & Nbrot & Nrizpl & ProdMF & ResMF & ProdMS & ResMS \\
\hline 1_1-1_2 & Não & Não & Não & Não & Não & Não & Não \\
\hline 1_1-2_1 & Sim & Não & Sim & Sim & Sim & Sim & Sim \\
\hline $1-1-2 \_2$ & Sim & Sim & Sim & Sim & Sim & Sim & Sim \\
\hline $1 \_2-2 \_1$ & Sim & Sim & Sim & Sim & Sim & Sim & Sim \\
\hline 1_2-2_2 & Sim & Sim & Sim & Sim & Sim & Sim & Sim \\
\hline $2 \_1-2 \_2$ & Não & Não & Não & Não & Não & Não & Não \\
\hline$p<0.05$ & $3,2 \mathrm{E}-05$ & $8,6 \mathrm{E}-05$ & 0,00 & $4,1 \mathrm{E}-05$ & $3,0 \mathrm{E}-05$ & 41E-05 & $3,24 \mathrm{E}-05$ \\
\hline
\end{tabular}

1_1=SAF simples replantada; $1 \_2$ = SAF simples espontânea; 2_1 = SAF biodiverso replantada e 2_2 = SAF biodiverso espontânea; Nbrot - número de brotações, Nrizpl - número de rizomas por planta, ProdMF - produção de rizomas em massa fresca, ResMF - resíduos em massa fresca, ProdMS - produção de rizomas em massa seca, ReMS - resíduos em massa seca.

Os resultados obtidos com estresse hídrico e térmico confirmam a rusticidade da araruta, que pode dispensar a necessidade do replantio e ocupar naturalmente o sub-bosque dos SAFs. Essa característica foi herdada no processo de seleção natural, sendo a araruta uma espécie nativa de ocorrência natural nas matas do Rio de Janeiro (FELTRAN; PERESSIN, 2014; MONTEIRO; PERESSIN, 2002). Para Bianchi et al. (2016) as plantas geófitas, como a araruta, que produzem rizomas usando o amido e carboidratos armazenados, são as mais adaptadas ao estresse hídrico. Para evitar a perda de água na parte aérea, a araruta reduz a superfície de exposição ao enrolar as folhas. Além disso, o reduzido crescimento vegetativo também é um mecanismo de escape à seca, pois, aumenta a capacidade da planta conduzir a água a curta distância. Com base nos resultados dessa pesquisa em ambientes com restrição hídrica e térmica momentânea a exploração bianual da araruta pode viabilizar a produção, para que as plantas tenham tempo para canalizar os fotoassimilados que serão convertidos em carboidratos nos drenos com a colheita realizada após um período com condições ambientais satisfatórias.

Dentre os fatores que justificam o cultivo da araruta, destacam-se a baixa demanda por tratos culturais e o baixo custo de implantação, que favorece a sua disseminação em regiões tropicais (SHINTU et al., 2015; ROHANDI et al., 2017); a possibilidade de manejo como planta espontânea em um modelo de exploração extensiva em SAFs focados na restauração ecológica; e a crescente tendência para se cultivar e consumir vegetais não convencionais (GUILHERME et al., 2017). Esses fatores fortalecem a conservação dessa espécie, que está em risco de extinção devido à indústria alimentícia ter substituído o polvilho de araruta pelo de mandioca, farinha de trigo ou milho, prejudicando, assim, o cultivo desta planta.

Em termos gerais, os experimentos de conversão agroflorestal melhoraram o aproveitamento da terra com a produção de culturas rústicas e adaptadas ao manejo agroflorestal. Para Altieri e Nicholls (2017), é importante iniciar o processo de transição para modelos agrícolas biodiversos com sistemas equilibrados e resilientes, que têm a possibilidade de superar as mudanças do clima, gerar renda com a produção de alimentos, fortalecer a segurança e a soberania alimentar, além de proteger a biodiversidade por meio da multifuncionalidade do sistema de produção agroecológica.

\section{CONCLUSÕES}

Os sistemas agroflorestais simples e biodiverso não prejudicam o crescimento do guanandi, que incrementa a circunferência a altura do peito no SAF simples após três anos de conversão agroflorestal.

$\mathrm{O}$ cultivo de mandioca e araruta tem desempenho compatível com a densidade de plantio utilizada nos SAFs, com produções superiores no SAF simples.

A araruta suporta o estresse hídrico e térmico em ambos os sistemas e dispensa o replantio ocupando naturalmente o subbosque dos SAFs.

O SAF simples é um sistema para agricultores interessados em obter o máximo rendimento de cultivos anuais em associação com a espécie florestal. O SAF biodiverso é adequado para agricultores que almejam diversificar a produção agrícola e restaurar o ambiente concomitantemente.

\section{REFERÊNCIAS}

ABDO, M. T.; MARTINS, A. L. M.; FINOTO, E. L.; FABRI, E. G.; PISSARRA, T. C. BIERAS, A. C.; LOPES, M. Implantação de sistema agroflorestal com seringueira, urucum e acerola sob diferentes manejos do Polo Regional Centro Norte/APTA. Pesquisa \& Teconologia, v.9, p.1-16, 2012.

ALTIERI, M. A.; NICHOLLS, C. I. The adaptation and mitigation potential of traditional agriculture in a changing climate. Climatic Change, v.140, n.1, p.33-45, 2017. 10.1007/s10584-013-0909-y

BASCHE, A. D.; EDELSON, O. F. Improving water resilience with more perennially based agriculture. Agroecology and Sustainable Food Systems, v. 41, n. 7, p. 799-824, 2017. $\underline{10.1080 / 21683565.2017 .1330795}$

BIANCHI, L.; GERMINO, G. H.; SILVA, M. A. Adaptação das plantas ao déficit hídrico. Acta Iguazu, v.5, n.4, p.15-32, 2016. 
BRANCALION, P. H. S.; VIANI, R. A. G.; RODRIGUES, R. R.; CÉSAR, R. G. Estratégias para auxiliar na conservação de florestas tropicais secundárias inseridas em paisagens alteradas. Ciências Naturais, v. 7, n. 3, p. 219-234, 2012.

BRENES, A. R.; MONTAGNINI F. Growth, productivity, aboveground biomass, and carbon sequestration of pure and mixed native tree plantations in the Caribean lowlands of Costa Rica. Forest Ecology and Management; v. 232, n. 1-3, p. 168178, 2006. 10.1016/j.foreco.2006.05.067

CARVALHO, P.E.R. Guanandi. 1 ed. Colombo: Embrapa Florestas, 2003. 14p. (Circular Técnica n. 78).

CASTRO, C. M.; DEVIDE, A. C. P.; VAllE, T. L.; FELTRAN, J. C.; MELO, T. J.; SANTANA, R. F. Desempenho de clones de mandioca em sistema orgânico. Revista Raízes e Amidos Tropicais, v.5, p. 375-381, 2009.

CIIAGRO - Centro Integrado de Informações Agrometeorológicas. Monitoramento agrometeorológico e climático. 2014.

DARONCO, C.; MELO, A. C. G.; MACHADO, J. A. R. Consórcio de espécies nativas da Floresta Estacional Semidecidual com mandioca (Manihot sculenta Crantz) para restauração de mata ciliar. Revista Árvore, v. 36, n. 2, p. 291 299, 2012. 10.1590/S0100-67622012000200010.

DEVIDE, A. C. P.; CASTRO, C. M. Mandioca: múltiplos usos na transição agroecológica. Pesquisa \& Tecnologia, v. 7, n. 2, 8p., 2010.

DEVIDE, A. C. P.; CASTRO, C. M.; VAlle, T. L.; FELTRAN, J. C.; MELO, T. J.; SANT'ANA, R. F. Competição de clones de mandioca de mesa no Vale do Paraíba paulista. Revista Raízes e Amidos Tropicais, v.5, p. 326-332, 2009.

DURIGAN, G.; SILVEIRA, E. R. da. Recomposição da mata ciliar em domínio de cerrado, Assis, SP. Scientia Forestalis, n. 56, p. 135-144, 1999.

FAO, Food and Agriculture Organization. Agroforestry for landscape restoration: Exploring the potential of agroforestry to enhance the sustainability and resilience of degraded landscapes. $1^{\text {a }}$ ed. Roma: FAO, 2017. 28p.

FAPESP, Fundação de Amparo à Pesquisa do Estado de São Paulo. Mandioca vitaminada. Revista FAPESP, v. 200, n.10, p.100-103, 2012.

FELTRAN J. C.; PERESSIN, V. A. Instruções agrícolas para as principais culturas econômicas. In: AGUIAR, A. T. da E.; GONÇALVES, C.; PATERNIANI, M. E. A. G. Z.; TUCCI, M. L. S.; CASTRO, C. E. de (eds.). Instruções agrícolas para as principais culturas econômicas. $7^{\mathrm{a}}$ ed. Campinas: Instituto Agronômico, 2014, p. 36-38 (Boletim 200).
GUILHERME, D. O.; REIS, L. K.; PRADO, N. B. F.; ARRUDA, M. W. G.; MORAIS, D. P.; CEREDA, M. P. Production arrowroot depending on the size of the rhizome and substrate in Campo Grande - MS, Brazil. Journal of Environmental \& Agriculture Research, v.3, n.1, p. 132-135, 2017.

KUMALASARI, I. D.; HARMAYANI, E.; LESTARI, L. A.; RAHARJO, S.; ASMARA, W.; NISHI, K.; SUGAHARA, T. Evaluation of immunostimulatory effect of the arrowroot (Maranta arundinacea L.) in vitro and in vivo. Cytotechnology, v. 64, n.2, p.131-137, 2012. 10.1007/s10616-011-9403-4

LAESTADIUS, L.; MAGINNIS, S.; MINNEMEYER, S; POTAPOV, P.; SAINT-LAURENT, C.; SIZER, N. Mapping opportunities for forest landscape restoration. Unasylva 238, v. 62, n. 2, p. 47-48, 2011.

LAUDARES, S. S. A.; BORGES, L. A. C.; ÁVILA, P. A.; OLIVEIRA, A. L.; SILVA, K. G.; LAUDARES, D. C. A. Agroforestry as a sustainable alternative for environmental regularization of rural consolidated occupations. CERNE, v. 23, n. 2, p. 161-174, 2017. 10.1590/01047760201723022240

LUEDELING, E.; ROELAND, K.; HUTH, N. I.; KOENING, $\mathrm{K}$. Agroforestry systems in a changing climate - challenges in projecting future performance. Current Opinion in Environmental Sustainability, v. 6, p. 1-7, 2014. $\underline{10.1016 / j . c o s u s t .2013 .07 .013}$

MACEDO, K. R. Utilização da parte aérea da Manihot esculenta Crantz na alimentação de frango de corte de linhagem caipira: revisão de literatura. Veterinária em Foco, v.13, n.2, p.76-86, 2016.

MEDEIROS, R. A.; PAIVA, H. N.; LEITE, H. G.; OLIVEIRA NETO, S. N.; VENDRÚSCOLO, D. G. S.; SILVA, F. T. Análise silvicultural e econômica de plantas clonais e seminais de Tectona grandis L.f. em sistema taungya. Revista Árvore, v. 39, n. 5, p. 893-903, 2015. 10.1590/0100-67622015000500012.

MONTEIRO, D. A.; PERESSIN, V. A. Cultura da araruta. In: CEREDA, M. P. (Coord.) Agricultura: tuberosas amiláceas Latino Americanas. São Paulo: Fundação Cargill, 2002, v. 2, p. 440-447.

MORENO, L. B.; TORALES, E. P.; HEID, D. M.; ZÁRATE, N. A. H.; ABRÃO, M. S. Influence of plant density and hilling on yield and profitability of arrowroot. Pesquisa Agropecuária Tropical, v. 47, n. 4, p. 465-471, 2017. 10.1590/1983$\underline{40632017 \mathrm{v} 4748643}$

ODEKU, O. A. Potentials of tropical starches as pharmaceutical excipients: A review. Starch, v. 65, n. 1-2, p. 89-106, 2013. $\underline{10.1002 / \text { star.201200076 }}$

PETIT, B.; MONTAGNINI, F. Growth in pure and mixed plantations of tree species used in reforesting rural areas of the humid region of Costa Rica, Central America. Forest Ecology 
and Management, v. 233, p. 338-343, 2006. 10.1016/j.foreco.2006.05.030

RAPPAPORT, D.; MONTAGNINI, F. Tree species growth under a rubber (Hevea brasiliensis) plantation: native restoration via enrichment planting in southern Bahia, Brazil. New Forests, v. 45, n. 5, p.715-732. 2014. 10.1007/s11056-0149433-9

ROHANDI, A.; BUDIADI; HARDIWINOTO, S.; HARMAYANI, E.; SUDRAJAT, D. J. Variability in morphophysiology, tuber yield and starch content of several arrowroot populations in Garut district. AGRIVITA Journal of Agricultural Science, v. 39, n. 3, 311-323, 2017. $\underline{10.17503 / a g r i v i t a . v 39 i 3.1002}$

SANTOS, M. J. C.; RODRIGUEZ, L. C. E.; WANDELLI, E. V. Avaliação econômica de quatro modelos agroflorestais em áreas degradadas por pastagens na Amazônia Ocidental. Scentia Forestalis, n. 62, p. 48-61, 2002.

SECRETARIA DO MEIO AMBIENTE. SÃO PAULO [Estado]. Oportunidades para restauração de paisagens e florestas na porção paulista do Vale do Paraíba: Plano de Desenvolvimento Florestal Territorial para a porção paulista do Vale do Paraíba. 1 ed. Porto Alegre : Ideograf, 2018, 213p.

SEIFERT S. N.; WATZL, B. Inulin and oligofructose: review of experimental data on immune modulation. The Journal of nutrition, v. 137, n. 11 Suppl, p. 2563S-2567S, 2007. $10.1093 / \mathrm{jn} / 137.11 .2563 \mathrm{~S}$
SHINTU, P.V.; RADHAKRISHNAN, V. V.; MOHANAN, K. V. Pharmacognostic standardisation of Maranta arundinacea L. - An important ethnomedicine. Journal of pharmacognosy and phytochemistry, v. 4, p. 242 - 246, 2015.

SILVA, H. R. F.; MELO, V. L.; PACHECO, D. D.; ASSIS, Y. J. M.; SALES, H. R. Acúmulo de matéria seca e micronutrientes em mandioca consorciada com bananeira. Pesq. Agropec. Trop., v. 44, n. 1, p. 15-23, 2014.

STATSOFT, INC. Statistica - Data analysis software system. Version 7.0.6.0. Tulsa, OK 74104, USA, 2004.

SWADIJA, O. K.; PADMNABHAN, V. B.; VIJAYARAGHAVA, K. Growth and yield of arrowroot intercropped in coconut garden as influenced by organic management. Journal of Root Crops, v. 39, n. 1, p. 67-72 67, 2013.

TARGA, M. S.; BATISTA, G. T. Benefits and legacy of the water crisis in Brazil. Rev. Ambient. Água, Taubaté, v. 10, n. 2, p. 234-239, 2015. 10.4136/ambi-agua.1629

ZÁRATE, N. A. H.; VIEIRA, M. C. Produção da araruta 'Comum' proveniente de três tipos de propágulos. Ciência e Agrotecnologia, v. 29, n. 5, p. 995-1000, 2005.

ZÁRATE, N. A. H.; VIEIRA, M. C.; HEID, D. M.; MORENO, L. B.; AQUINO, V. M.; MIYASHIRO, M. R. Produtividade da araruta 'Comum' cultivada com diferentes espaçamentos entre plantas e número de fileiras no canteiro. Horticultura Brasileira, v. 30, p. S3573-S3579, 2012. 